\title{
Free-Positioning Wireless Power Transfer Using a 3D Transmitting Coil for Portable Devices
}

\author{
Nam Ha-Van ${ }^{1} \cdot$ Hoang Le-Huu ${ }^{1} \cdot$ Minh Thuy Le ${ }^{2} \cdot K_{w a n g s u k ~ P a r k}{ }^{3} \cdot$ Chulhun Seo ${ }^{1, *}$
}

\begin{abstract}
The free-positioning wireless power transfer (WPT) system has drawn attention in recent years. Traditionally, a WPT system can transfer energy in one or two directions on the same plane, but it leads the restrictions of angle and axis misalignment between a transmitter and a receiver coil. In this paper, we propose a free-positioning WPT system using a three-dimensional cubic-shaped transmitting coil for portable device charging. A small receiving coil is placed inside the transmitter to achieve the transferred energy through the magnetic resonant coupling. In addition, the equivalent circuit and the mutual inductance between the $\mathrm{Tx}$ and $\mathrm{Rx}$ coils are analyzed. Finally, a practical experiment is implemented to verify the transfer performance, which can reach up to about $50 \%$ power transfer efficiency. The proposed system can charge in spatial freedom.
\end{abstract}

Key Words: Cubic-Shaped, Free-Positioning, Magnetic Resonant Coupling, Wireless Power Transfer (WPT).

\section{INTRODUCTION}

Wireless power transfer (WPT) has attracted much attention because of its potential application in consumer electronic products, portable devices, robotics, electric vehicles, and charging systems [1-6]. With the occurrence of portable device explosion, the demand for wireless charging is necessary to reduce the mass of the traditional wire charge system. The WPT system for portable devices has some restrictions. A conventional WPT system still suffers from efficiency degradation due to changes in the position of the receiver, such as the variations in the distance, angle, and axial misalignment. For convenient wireless charging and for overcoming the restrictions of the conventional WPT system, free positioning is necessary to create reliable wireless charging systems and maintain the power transfer efficiency
(PTE). In recent research, the free-positioning WPT has been explored in comparison with the use of one direction or two directions on the same plane [7-9]. In [10], a WPT system using a bowl-shaped transmitting (Tx) coil for free-positioning is proposed for charging small electronic devices, such as hearing aids and wearable electronic devices with a rechargeable battery. However, the bowl-shaped coil presents a restriction when the small receiver $(\mathrm{Rx})$ coil is in the perpendicular arrangement. Therefore, the mutual inductances between the Tx and Rx resonant coils are small, leading to a low performance of the system. Moreover, a resonant WPT system that allows the freepositioning of a wearable device at a $360^{\circ}$ axis of rotation and the simultaneous charging of a mobile phone is proposed in [11]. The smartwatch is placed at a small fixed distance around the cylindrical charging surface. The application of this structure

Manuscript received April 15, 2020 ; Revised June 9, 2020 ; Accepted July 16, 2020. (ID No. 20200415-048J)

${ }^{1}$ Department of Information Communication, Materials, and Chemistry Convergence Technology, Soongsil University, Seoul, Korea.

${ }^{2}$ Department of Instrument and Industrial Informatics, School of Electrical Engineering, Hanoi University of Science and Technology, Hanoi, Vietnam.

${ }^{3}$ Department of Biomedical Engineering, Seoul National University College of Medicine, Seoul, Korea.

"Corresponding Author: Chulhun Seo (e-mail: chulhun@ssu.ac.kr)

This is an Open-Access article distributed under the terms of the Creative Commons Attribution Non-Commercial License (http://creativecommons.org/licenses/by-nc/4.0) which permits unrestricted non-commercial use, distribution, and reproduction in any medium, provided the original work is properly cited.

(c) Copyright The Korean Institute of Electromagnetic Engineering and Science. All Rights Reserved. 
is quite specific and can charge a smartwatch and a mobile phone.

In [12, 13], a multi-directional magnetic resonant coupling WPT is presented using a cubic Tx design. A receiver $(\mathrm{Rx})$ is placed outside of the Tx coil, and the spatial freedom of the $\mathrm{Rx}$ coil is not developed. In the current study, a WPT system with an $\mathrm{Rx}$ coil placed inside the cubic-shaped Tx coil is proposed for free-positioning. The WPT system is a two-coil WPT system that is compact and suitable for consumer applications compared with the four-coil WPT system, such as portable and wearable device chargers, mobile phones, and tablets. A circuit analysis of the proposed system is presented to obtain the maximum PTE. The system is simulated using Ansoft HFSS software and fabricated to evaluate performance.

This paper is organized as follows. The magnetic field of a $\mathrm{Tx}$ coil is evaluated and analyzed for free-positioning charging in Section II. The sceneries of the Rx positions inside the Tx coil are demonstrated to validate the spatial freedom charging. In Section III, the Tx and Rx coils are fabricated and measured to evaluate the efficiencies. The conclusion is presented in Section IV.

\section{FREE-POSITIONING WPT ANALYSIS}

In general, WPT through magnetic resonant coupling is experimentally demonstrated in a system with two resonant loop antennas in one or two directions on the same plane. A conventional WPT system suffers a misalignment between the Tx coil and the Rx coil on the planar charging platform. A coil designed with different geometries can modify the radiation pattern, which overcomes the inconvenience by increasing the direction of mutual coupling. A cubic-shaped Tx coil is proposed for free-positioning wireless charging with a small $\mathrm{Rx}$ coil that remains in spatial freedom. The purpose is to overcome the limitations of the conventional system.

\section{WPT System with a 3D Transmitting Coil}

Fig. 1 shows the Tx coil structure and the magnetic field vector generated by the driven current on each loop side of the $\mathrm{cu}^{-}$ bic-shaped Tx coil [12]. The cubic Tx is constructed by folding the copper wire along the edges of the cube. The folding wire should be positioned in such a way that the current is driven as a closed-loop on each side of the cube, as shown in Fig. 1(a). In the first half of the period, the current follows counter-clockwise, resulting in the directions of the magnetic field to be perpendicular to each side of the cube and vice-versa using the right-hand screw rule, which is expressed as $\overrightarrow{H_{1}} \sim \overrightarrow{H_{6}}$. To verify the theoretical analysis, the cubic- shaped Tx structure is simulated to plot the magnetic field at the operating frequency of $13.56 \mathrm{MHz}$ band for consumer electronics to avoid the need for frequency

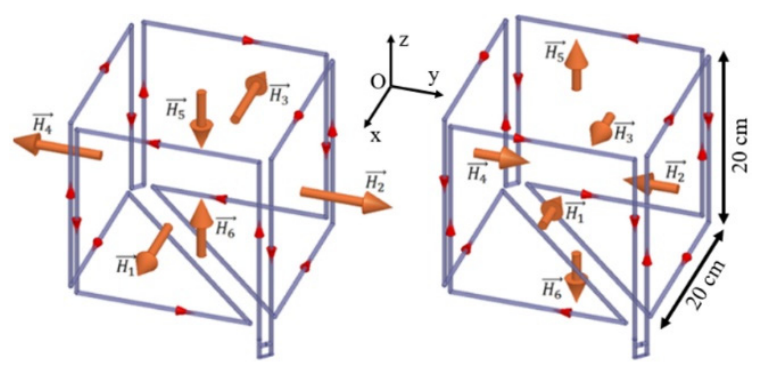

(a)
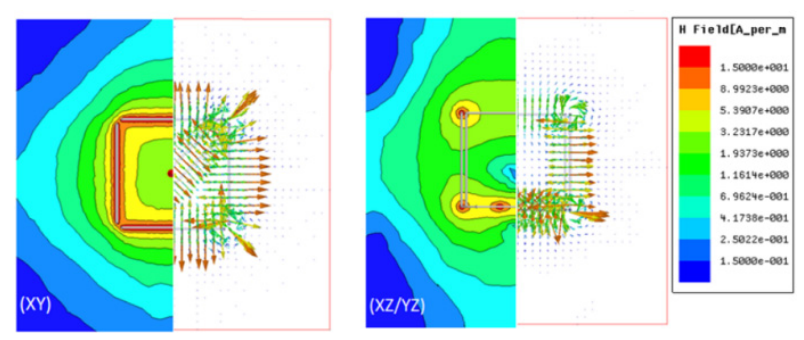

(b)

Fig. 1. The cubic-shaped Tx coil structure: (a) magnetic field vector in each of the half-operating period $\mathrm{T} / 2$ and (b) magnetic field simulation with its magnetic field density and magnetic field vector.

allocation. With this operating frequency, the wavelength of the current is much larger than the circuit dimension. Therefore, we can assume that the driven current in each side loop of the $\mathrm{Tx}$ coil is identical and in phase. The magnetic field inside the $\mathrm{Tx}$ coil is omnidirectional and has a significantly strong magnitude, as shown in Fig. 1(b). The snapshots of the simulated distributions of the magnetic field vectors generated by the Tx coil agree well with the theoretical analysis in the above section. The magnetic vectors are perpendicular to the loop sides and emitted at the corners of the cubic Tx coil. Therefore, a small $\mathrm{Rx}$ coil can be put inside the $\mathrm{Tx}$ coil to receive the energy from magnetic resonant coupling.

\section{Mutual Inductance Calculation}

Fig. 2(a) shows the schematic diagram of the system for mutual inductance calculation, with the Rx position determined by the rotation angles $\varphi$ and $\theta$ and the movement parameters $d_{x}, d_{y}$, and $d_{z}$. To derive the mutual inductance between two coils, the Tx and Rx structures are divided into multiple pieces. Each $\mathrm{Tx}$ and $\mathrm{Rx}$ coil pieces are represented by a vector $\vec{u}_{i}\left(x_{i}, y_{i}, z_{i}\right)$ and $\vec{u}_{j}\left(x_{j}, y_{j}, z_{j}\right)$, respectively, and the vector direction is decided by the current direction in the corresponding coil. The transformation vector coordinates $\vec{u}_{i}\left(x_{i}, y_{i}, z_{i}\right)$ and ${\overrightarrow{u_{j}^{\prime}}}_{j}\left(x_{j}^{\prime}, y_{j}^{\prime}, z_{j}^{\prime}\right)$ of the Rx coil are determined by

$$
\begin{gathered}
x_{j}^{\prime}=x_{j} \cos \varphi \cos \theta+y_{j}(\cos \varphi \sin \theta-\sin \varphi) \\
+z_{j} \cos \varphi \sin \theta+d_{x} \\
y_{j}^{\prime}=x_{j} \sin \varphi \cos \theta+y_{j} \cos \varphi \cos \theta-z_{j} \cos \theta \sin \varphi+d_{y}
\end{gathered}
$$




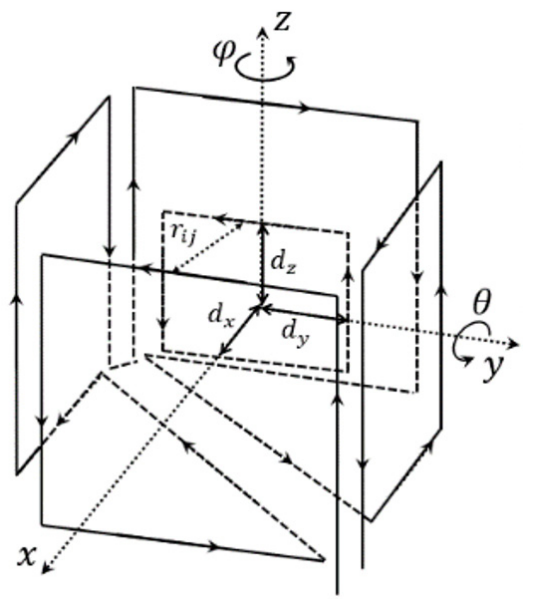

Fig. 2. (a) Schematic diagram of the free-positioning WPT system; the Tx and Rx coils.

$$
z_{j}^{\prime}=-x_{j} \sin \theta+z_{j} \cos \theta+d_{z}
$$

The overall mutual inductance between the Tx and Rx coils is obtained by the summation of the individual mutual inductances between two arbitrary coil pieces:

$$
\begin{aligned}
& M_{\overline{12}}=\frac{\mu_{0}}{4 \pi} \sum_{i=1}^{N_{1}} \sum_{j=1}^{N_{2}} \frac{\mu_{0}}{4 \pi} \frac{\vec{u}_{i} \cdot \overrightarrow{u_{j}^{\prime}}}{r_{i j}} \\
& =\frac{\mu_{0}}{4 \pi} \sum_{i=1}^{N_{1}} \sum_{j=1}^{N_{2}} \frac{x_{i} x_{j}^{\prime}+y_{i} y_{j}^{\prime}+z_{i} z_{j}^{\prime}}{\sqrt{\left(x_{i}-x_{j}^{\prime}\right)^{2}+\left(y_{i}-y_{j}^{\prime}\right)^{2}+\left(z_{i}-z_{j}^{\prime}\right)^{2}}}
\end{aligned}
$$

where $N_{1}$ and $N_{2}$ are the number of discrete segments in the Tx coil and Rx coil, respectively, and $\mu_{0}$ is the permeability of the free-space medium.

A comparison of mutual inductance between the calculated and simulated results is plotted in Fig. 2(b). There is a good agreement between the simulated and the calculated results obtained using (4). Owing to the cancellation of the magnetic field in the center region, the mutual inductances between the $\mathrm{Tx}_{\mathrm{x}}$ and $\mathrm{Rx}$ coils are conducive to the low values in this area. The mutual inductances at the receiving angle $\theta$ are higher than the others because there are two diagonally folded wires at the bottom of the cubic Tx coil.

\section{Circuit Model and System Analysis}

Fig. 3(a) shows the sketch of the WPT system with the $\mathrm{Rx}$ coil placed inside the $T_{x}$ structure. The volume of the $T_{x}$ coil is $20 \mathrm{~cm} \times 20 \mathrm{~cm} \times 20 \mathrm{~cm}$, and the dimension of the $\mathrm{Rx}$ coil is $10 \mathrm{~cm} \times 10 \mathrm{~cm}$. Both the $\mathrm{Tx}$ and Rx coils are made with copper wire with a diameter of $5 \mathrm{~mm}$. Fig. 3(b) shows the equivalent circuit model of the proposed WPT system to analyze the transfer efficiency. The schematic is composed of two resonant circuits corresponding to the $\mathrm{Tx}_{\mathrm{x}}$ and $\mathrm{Rx}$ coils. By using a series capacitor and a shunt capacitor, the coil impedance can be

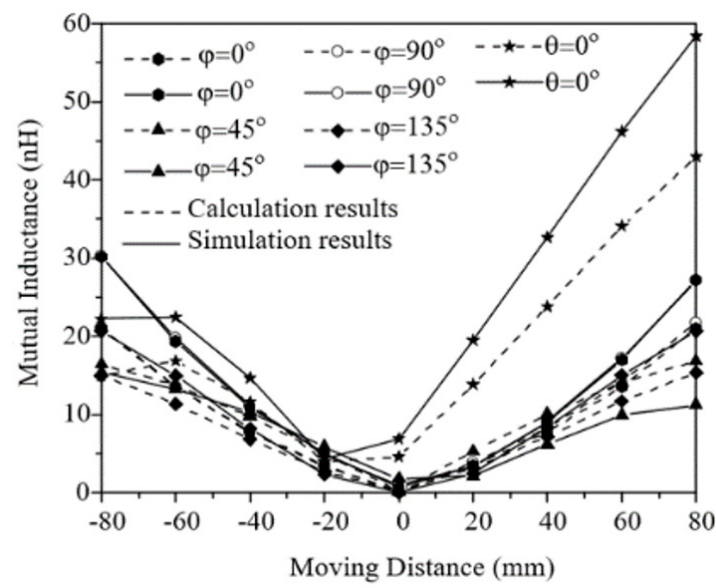

(b) calculated and simulated results of the mutual inductance between

(1)

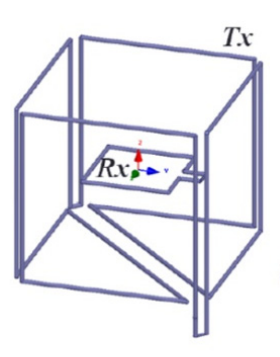

(a)

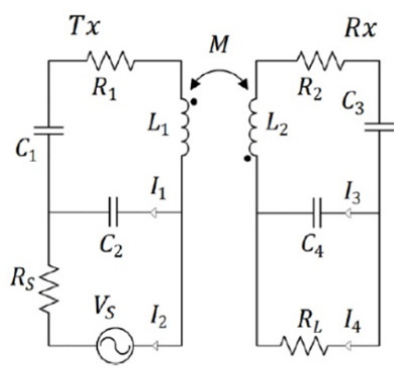

(b)
Fig. 3. (a) Free-positioning WPT system in a 3D view. (b) Equivalent circuit model of the resonant coupling two-coil system, which is composed of lumped components.

matched with almost all load impedances [13]. Kirchhoffs voltage law (KVL) is used to determine the currents as presented by

$$
\begin{gathered}
\left(I_{1}+I_{2}\right) Z_{1}+j \omega M\left(I_{3}+I_{4}\right)+I_{2} R_{S}=V_{S} \\
\left(I_{3}+I_{4}\right) Z_{2}+j \omega M\left(I_{1}+I_{2}\right)+I_{4} R_{L}=0
\end{gathered}
$$

where

$$
\begin{gathered}
Z_{1}=R_{1}+j \omega L_{1}+\frac{1}{j \omega C_{1}} \\
Z_{2}=R_{2}+j \omega L_{2}+\frac{1}{j \omega C_{3}} \\
V_{S}=I_{2} R_{S}-\frac{I_{1}}{j \omega C_{2}} \\
V_{L}=-R_{L} I_{4}=-\frac{I_{3}}{j \omega C_{4}} .
\end{gathered}
$$

The KVL equations are derived to explore the transfer function $V_{L} / V_{S}$ :

$$
\begin{gathered}
\frac{V_{L}}{V_{S}}=\frac{j \omega k_{\varphi} \sqrt{L_{1} L_{2}} R_{L}}{\omega^{4} k_{\varphi}^{2} L_{1} L_{2} C_{2} C_{4} R_{S} R_{L}-j \omega^{3} k_{\varphi}^{2} L_{1} L_{2}\left(C_{2} R_{S}+C_{4} R_{L}\right)} \\
-\omega^{2}\left(k_{\varphi}^{2} L_{1} L_{2}-C_{2} C_{4} R_{S} R_{L} Z_{1} Z_{2}\right)-j \omega\left[C _ { 2 } R _ { S } Z _ { 1 } \left(R_{L}\right.\right. \\
\left.\left.\quad+Z_{2}\right)+C_{4} R_{L} Z_{2}\left(R_{S}+Z_{1}\right)\right]-\left(R_{S}+Z_{1}\right)\left(R_{L}+Z_{2}\right) .
\end{gathered}
$$


The PTE is calculated according to the $S_{21}$ by PTE $=$ $\left|S_{21}\right|^{2} \times 100(\%)$. The equivalent $\left|S_{21}\right|$ scattering parameter can be calculated by

$$
\left|S_{21}\right|=2\left|\frac{V_{L}}{V_{S}}\right| \sqrt{\frac{R_{S}}{R_{L}}}
$$

Fig. 4 illustrates the receiving positions of the Rx coil corresponding to the receiving angles $\varphi, \theta$. With each receiving angle, the $\mathrm{Rx}$ coil is moved around the origin of the coordinate. By adjusting the Rx's positions inside the space of the Tx coil, the PTEs of the system are verified, demonstrating the feasibility of the free-positioning wireless charging of the proposed system. The fabrication and experiment of the WPT system are presented in the following section.

\section{FABRICATION AND EXPERIMENTAL VALIDATION}

\section{OF THE WPT SYSTEM}

To verify the theoretical analysis, the resonant coupling system is constructed using a long copper wire with a radius of 2.5 $\mathrm{mm}$ for both the Tx and Rx coils. The total length of the copper wire for the cubic Tx coil is approximately $870 \mathrm{~cm}$. The lengths of the edge sides of the Tx coil are the same at $20 \mathrm{~cm}$. The real volume of the cubic Tx coil is $20.6 \mathrm{~cm} \times 20.8 \mathrm{~cm} \times$ $20.5 \mathrm{~cm}$. The dimension of the Rx coil is $10 \mathrm{~cm} \times 10 \mathrm{~cm}$. To determine the working frequency, each coil is tested separately with a Protek A333 network analyzer (GS Instech, Incheon, Korea). The measured resonant frequencies of the $\mathrm{Tx}$ and $\mathrm{Rx}$ coils are $13.586 \mathrm{MHz}$ and $13.574 \mathrm{MHz}$, respectively. The detailed values of the lumped elements and extracted parasitic components of the Tx and Rx coils are listed in Table 1. Fig. 5 illustrates the configuration of the free-positioning WPT system using a Protek A333 network analyzer.

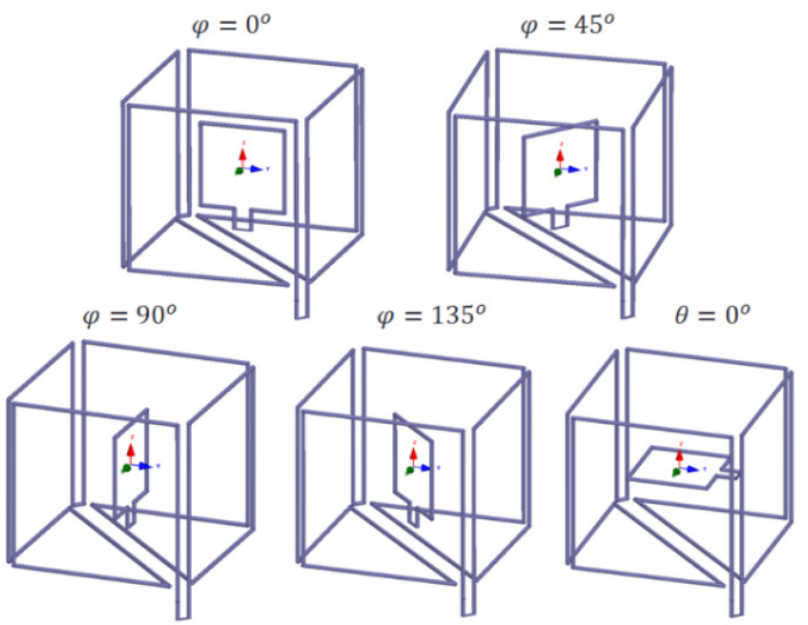

Fig. 4. Cubic-shaped Tx coil structure with the Rx coil positions corresponding to the receiving angles $\varphi, \theta$.
Table 1. Extracted component values of the Tx and Rx coils

\begin{tabular}{ccccc}
\hline \multicolumn{2}{c}{ Tx coil } & & & \multicolumn{2}{c}{ Rx coil } \\
\cline { 1 - 2 } \cline { 5 - 6 } Parameter & Value & & Parameter & Value \\
\hline$R_{S}$ & $50 \Omega$ & & $R_{L}$ & $50 \Omega$ \\
$R_{1}$ & $0.59 \Omega$ & & $R_{2}$ & $0.31 \Omega$ \\
$C_{1}$ & $81 \mathrm{pF}$ & & $C_{3}$ & $256 \mathrm{pF}$ \\
$C_{2}$ & $470 \mathrm{pF}$ & & $C_{4}$ & $910 \mathrm{pF}$ \\
$L_{1}$ & $1.9 \mu \mathrm{H}$ & & $L_{2}$ & $0.4 \mu \mathrm{H}$ \\
$Q_{1}$ & 274 & & $Q_{2}$ & 110 \\
\hline
\end{tabular}

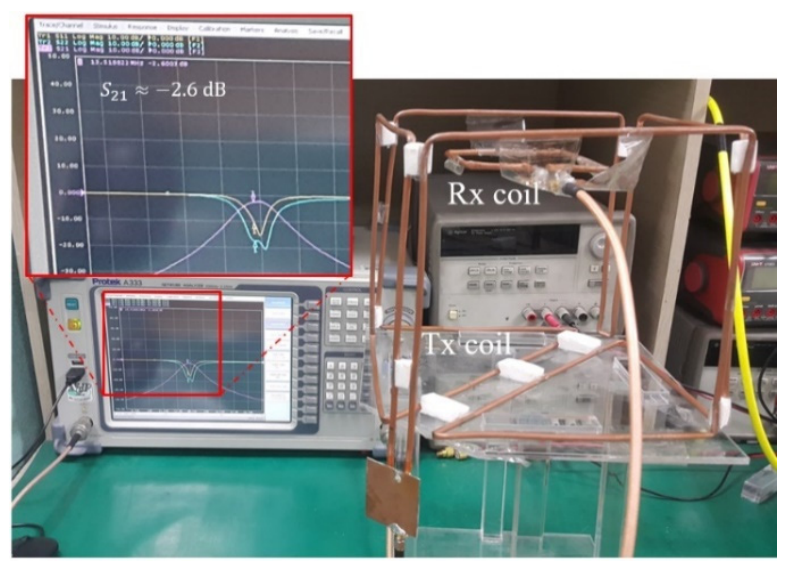

Fig. 5. Measurement configuration of the resonant coupling system. Ports 1 and 2 of the Network Analyzer Protek A333 are connected to the $\mathrm{Tx}$ and $\mathrm{Rx}$ coils, respectively.

Once the Tx and Rx parameters and their working frequencies are established, the Rx coil is displaced up to $8 \mathrm{~cm}$ from the origin of the coordinate system inside the cubic Tx coil. Alternatively, the PTE of the WPT system can be measured as the ratio of the output power to the input power. A signal generator (Agilent E4436B; Agilent Technologies Inc., Santa Clara, CA, USA) is used to supply the input power to the Tx coil, and while a power meter is used to measure the delivered power of the $\mathrm{Rx}$ coil by a spectrum analyzer (Agilent $85665 \mathrm{EC}$ ). The signal generator has a maximum generating power of $19 \mathrm{dBm}$. To extend the input power range, a $+45-\mathrm{dBm}$ drive amplifier is added to a transmitting module. The spectrum analyzer has a measured limitation of $30 \mathrm{dBm}$. Therefore, $\mathrm{a}-40-\mathrm{dB}$ attenuator is used to reduce the power transferred to the spectrum analyzer. The experimental setup of the WPT system is shown in Fig. 6.

In this experiment, the generating $\mathrm{Tx}$ coil is maintained in a fixed position, and the Rx coil is arranged inside the Tx coil at various receiving angles. The PTEs of the WPT system are illustrated in Fig. 7.

The Rx coil receives the highest energy at a distance of $4 \mathrm{~cm}$ from the origin of the coordinate system. In the small middle area of the Tx coil, the magnetic field is canceled because of 
Table 2. Comparison of the proposed system with those of related works

\begin{tabular}{lcccc}
\hline & Kim et al. [10] & Zhang et al. [14] & Liang et al. [15] & This work \\
\hline Structure & Bowl-shaped & Helmholtz coil & Triangular & Cubic \\
Characteristic & Dead zone & No dead zone & Ununiform free-positioning & Dead zone \\
Available arrangement & Parallel & Parallel & Parallel and perpendicular & Parallel and perpendicular \\
PTE for one load & Overall 30\% & Overall 55\% & Coil-to-coil 26.6\% & Coil-to-coil 50\% \\
\hline
\end{tabular}

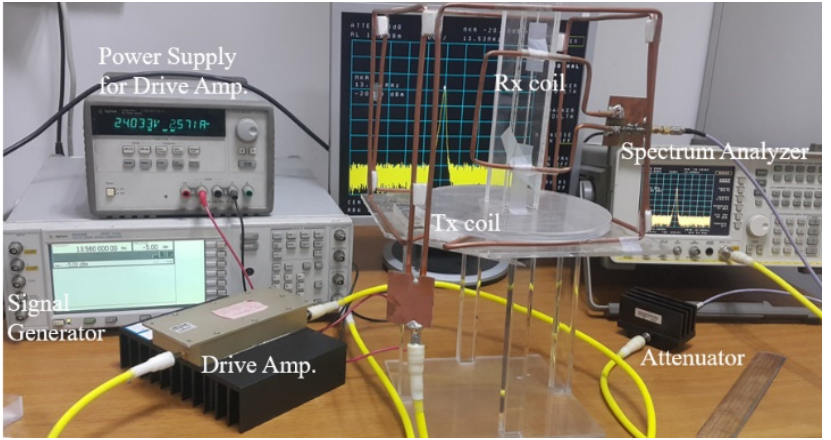

Fig. 6. Experiment setup of the WPT system.

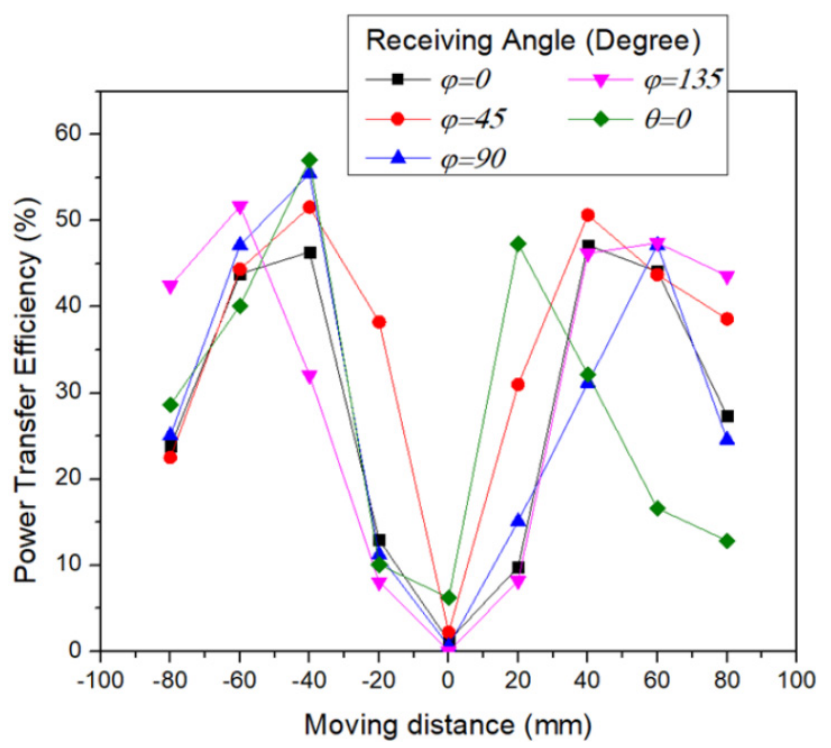

Fig. 7. Dependence between the PTE and the resonant coil distance. The distance is from the origin of the coordinate system to the Rx coil.

symmetric construction.

Therefore, the PTEs are almost zero in the middle position leading to the dead zone of the Tx structure. However, the effective area inside the $\mathrm{Tx}$ coil is dominated in the small dead zone, which occupies over $62 \%$ of the spatial area. The $\mathrm{Rx}$ coil is freely placed in parallel and perpendicular arrangements. At effective distances, the PTEs can achieve 50\%-60\% at the operating frequency of $13.56 \mathrm{MHz}$ at almost receiving angles. Table 2 shows a comparison of the structure types, system characteristics, possible arrangement, and performance of this study with those of previous research. Almost structures present some re- strictions for wireless charging, such as dead zone existence, limited arrangement, and incomplete spatial freedom charging. The proposed cubic Tx coil still contains a small dead zone in the central region inside the structure. However, the $\mathrm{Tx}$ coil presents a compatible high PTE for the free-positioning WPT system, with the free placement of the $\mathrm{Rx}$ coil in parallel and perpendicular arrangements except for the narrow dead zone.

\section{CONCLUSION}

We presented a cubic Tx coil design for the free-positioning magnetic resonant coupling WPT system. The proposed Tx coil could deliver power to the $\mathrm{Rx}$ coil arranged inside it with an efficiency of $50 \%$ at an operating frequency of $13.56 \mathrm{MHz}$ and an optimum distance of $4 \mathrm{~cm}$. The dependence between PTE and distance was investigated to validate the performance of the WPT system. In conclusion, low-cost fabrication without the current control methodology was implemented to verify the practical design of a cubic Tx coil with a simple single $\mathrm{Rx}$ coil.

This work was supported by the National Research Foundation of Korea (NRF) grant funded by the Korean government (MSIP) (No. NRF-2017R1A5A1015596) and by the Ministry of Education and Training (Project No. B2020BKA-11).

\section{REFERENCES}

[1] A. Kurs, A. Karalis, R. Moffatt, J. D. Joannopoulos, P. Fisher, and M. Soljacic, "Wireless power transfer via strongly coupled magnetic resonances," Science, vol. 317, no. 5834, pp. 8386, 2007.

[2] H. Hoang, S. Lee, Y. Kim, Y. Choi, and F. Bien, "An adaptive technique to improve wireless power transfer for consumer electronics," IEEE Transactions on Consumer Electronics, vol. 58, no. 2, pp. 327-332, 2012.

[3] J. Park, J. Kim, Y. Shin, B. Park, W. S. Kim, S. J. Cheong, and S. Ahn, "Toroidal-shaped coils for a wireless power transfer system for an unmanned aerial vehicle," Journal of Electromagnetic Engineering and Science, vol. 19, no. 1, pp. 48-55, 
2019.

[4] W. Huan, and H. Ku, "Analysis and optimization of wireless power transfer efficiency considering the tilt angle of a coil," Journal of Electromagnetic Engineering and Science, vol. 18, no. 1, pp. 13-19, 2018.

[5] Z. N. Low, R. A. Chinga, R. Tseng, and J. Lin, "Design and test of a high-power high-efficiency loosely coupled planar wireless power transfer system," IEEE Transactions on Industrial Electronics, vol. 56, no. 5, pp. 1801-1812, 2009.

[6] T. P. Duong and J. W. Lee, "Experimental results of highefficiency resonant coupling wireless power transfer using a variable coupling method," IEEE Microwave and Wireless Components Letters, vol. 21, no. 8, pp. 442-444, 2011.

[7] A. P. Sample, D. A. Meyer, and J. R. Smith, "Analysis, experimental results, and range adaptation of magnetically coupled resonators for wireless power transfer," IEEE Transactions on Industrial Electronics, vol. 58, no. 2, pp. 544-554, 2011.

[8] B. 1. Cannon, J. F. Hoburg, D. D. Stancil, and S. C. Goldstein, "Magnetic resonant coupling as a potential means for wireless power transfer to multiple small receivers," IEEE Transactions on Power Electronics, vol. 24, no. 7, pp. 1819-1925, 2009.

[9] N. Ha-Van, H. Le-Huu, and C. Seo, "Design of freepositioning wireless power transfer using a half-rectangular prism transmitting coil," in Proceedings of 2019 IEEE Wireless Power Transfer Conference (WPTC), London, UK, 2019, pp. 85-88.

[10] J. Kim, D. H. Kim, J. Choi, K. H. Kim, and Y. J. Park,

$\mathrm{Nam} \mathrm{Ha-Van}$

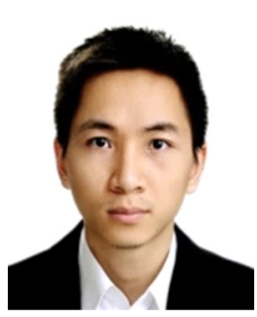

received his B.S. degree from the School of Electronics and Telecommunications, Hanoi University of Science and Technology, Hanoi, Vietnam, in 2012, and his $\mathrm{Ph} . \mathrm{D}$. degree in information and telecommunication engineering from Soongsil University, Seoul, South Korea, in 2019, where he is currently a postdoctoral researcher at the Soongsil University Foundation of University Industry Cooperation. His current research interests include wireless power transfer, metamaterials, power amplifiers, phased array antennas, and energy harvesting systems.
"Free-positioning wireless charging system for small electronic devices using a bowl-shaped transmitting coil," IEEE Transactions on Microwave Theory and Techniques, vol. 63, no. 3, pp. 791-800, 2015.

[11] R. C. Kuo, P. Riehl, A. Satyamoorthy, W. Plumb, P. Tustin, and J. Lin, "A 3D resonant wireless charger for a wearable device and a mobile phone," in Proceedings of 2015 IEEE Wireless Power Transfer Conference (WPTC), Boulder, CO, 2015.

[12] N. Ha-Van and C. Seo, "A novel cubic transmitter for multi-directional wireless power transfer," in Proceedings of 2015 IEEE Wireless Power Transfer Conference (WPTC), Boulder, CO, 2015.

[13] N. Ha-Van and C. Seo, "Analytical and experimental investigations of omnidirectional wireless power transfer using a cubic transmitter," IEEE Transactions on Industrial Electronics, vol. 65, no. 2, pp. 1358-1366, 2018.

[14] W. Zhang, T. Zhang, Q. Guo, L. Shao, N. Zhang, X. Jin, and J. Yang, "High-efficiency wireless power transfer system for 3D, unstationary free-positioning and multi-object charging," IET Electric Power Applications, vol. 12, no. 5, pp. 658-665, 2018.

[15] Z. Liang, J. Wang, Y. Zhang, J. Jiang, Z. Yan, and C. Mi, "A compact spatial free-positioning wireless charging system for consumer electronics using a three-dimensional transmitting coil," Energies, vol. 12, no. 8, article no. 1409, 2019.

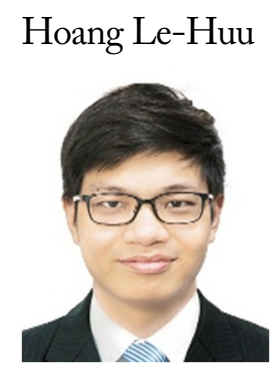

received his B.S. degree from the School of Electronics and Telecommunication, Hanoi University of Science and Technology, Hanoi, Vietnam, in 2018. He is currently pursuing his Ph.D. degree in information and telecommunication engineering at Soongsil University, Seoul, South Korea. His current research interests include wireless power transfer, antenna design, and computational electromagnetics. 


\section{Minh Thuy Le}

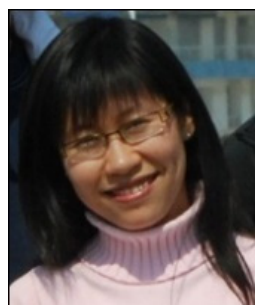

received he B.S. and M.S. degrees in electrical engineering from Hanoi University of Science and Technology, Hanoi, Vietnam, in 2006 and 2008, respectively, and her $\mathrm{Ph} . \mathrm{D}$. degree in optical and radio frequency from Grenoble Institute of Technology, France, in 2013. She is a lecturer and group leader of the radio frequency group at the Department of Instrumentation and Industrial Informatic (3I), School of Electrical Engineering, Hanoi University of Science and Technology. Her research interests include indoor localization, antenna, metamaterials, RF energy harvesting, and wireless power transfer.

\section{Chulhun Seo}

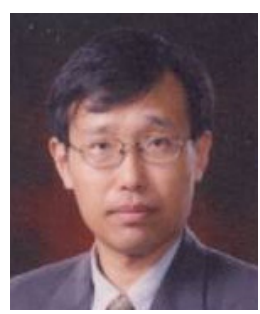

received his B.S., M.S., and Ph.D. degrees from Seoul National University, Seoul, South Korea, in 1983, 1985, and 1993, respectively. From 1993 to 1995, he worked at the Massachusetts Institute of Technology, Cambridge, MA, USA, as a technical staff member. From 1993 to 1997, he worked as an assistant professor at Soongsil University, Seoul, Korea. From 1999 to 2001, he was a visiting professor at MIT. From 1997 to 2004, he served as an assistant professor at Soongsil University, where he has been a professor of electronic engineering since 2004. He served as the IEEE MTT Korea Chapter Chairman, from 2011 to 2014. He is the Director of the Wireless Power Transfer Research Center supported by the Korean Ministry of Trade, Industry, and Energy, and the Director of Metamaterials Research Center, which is supported by the Basic Research Laboratories through the NRF Grant funded by the MSIP. His research interests include wireless communication technologies, RF power amplifiers, and wireless power transfer using metamaterials.
Kwangsuk Park

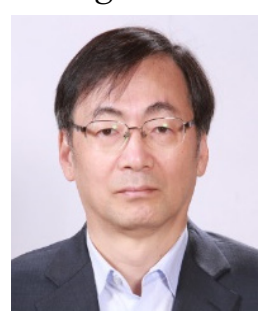

received his B.S. and Ph.D. degrees from the Department of Electronics Engineering in Seoul National University, Korea, in 1980 and 1985, respectively. He is currently a professor at the Department of Biomedical Engineering at Seoul National University and has been since he joined the department in 1985 as a founding staff member. He is a member of the Korean Society of Medical and Biological Engineering and has served as the president in 2014. He also served as the secretary general of the World Congress on Medical Physic and Biomedical Engineering held in Seoul in 2006. He is a senior member of IEEE EMBS and served as an associate editor of IEEE Journal of Biomedical and Health Informatics from 2006 to 2012 . He served as the conference chair in EMBC2017. He has chaired and co-chaired the annual International Conference on $\mathrm{u}-\mathrm{Healthcare}$ in the last 14 years. His main research area is biological signal measurement and processing for diagnosis. Currently, he is focusing his research interest on nonintrusive measurements for ubiquitous healthcare. 